\title{
CD80 (B7.1) and CD86 (B7.2) induce EBV-transformed $B$ cell apoptosis through the Fas/FasL pathway
}

\author{
GA BIN PARK ${ }^{1}$, YEONG SEOK KIM ${ }^{1}$, HYUN-KYUNG LEE ${ }^{2}$, \\ DAE-HO CHO ${ }^{3}$, DAEJIN KIM ${ }^{1}$ and DAE YOUNG HUR ${ }^{1}$ \\ ${ }^{1}$ Department of Anatomy and Research Center for Tumor Immunology, Inje University College of Medicine; \\ ${ }^{2}$ Department of Internal Medicine, Inje University Busan Paik Hospital, Busan 614-735; ${ }^{3}$ Department of Life Science, \\ Sookmyung Women's University, Yongsan-gu, Yongsan-ku, Seoul 140-742, Republic of Korea
}

Received July 7, 2013; Accepted August 16, 2013

DOI: 10.3892/ijo.2013.2091

\begin{abstract}
CD80 and CD86 expression is strongly regulated in $\mathrm{B}$ cells and is induced by various stimuli (e.g., cytokines, ligation of MHC class II and CD40 ligand). Epstein-Barr virus (EBV) infection activates B lymphocytes and transforms them into lymphoblastoid cells. However, the role of CD80 and CD86 in EBV infection of B cells remains unclear. Here, we observed that cross-linking of CD80 and CD86 in EBV-transformed $\mathrm{B}$ cells induced apoptosis through caspase-dependent release of apoptosis-related molecules, cytochrome $c$ and apoptosisinducing factor (AIF) from mitochondria, because Z-VAD-fmk ( $N$-benzyloxycarbonyl-Val-Ala-Asp-fluoromethylketone) and $\mathrm{N}$-acetylcysteine (NAC) blocked apoptosis and disruption of mitochondria. Stimulation of CD80 and CD86 induced expression of Fas ligand (FasL) on EBV-transformed B cells and upregulated Fas and FasL expression in IM-9 cells. Apoptosis through Fas-FasL interactions was blocked by treatment of cells with ZB4, an antagonistic anti-Fas antibody. These results suggest that the co-stimulatory molecules CD80 and CD86 induced by EBV infection stimulate apoptosis of EBV-transformed lymphoblastoid B cells via the Fas/FasL pathway.
\end{abstract}

\section{Introduction}

Epstein-Barr virus (EBV) is a member of the ubiquitous human $\gamma$ herpes virus family and $\sim 90 \%$ of the world-wide population is thought to be infected (1). In vitro infection of

Correspondence to: Professor Dae Young Hur or Professor Daejin Kim, Department of Anatomy and Research Center for Tumor Immunology, Inje University College of Medicine, Kaekum-2-dong, Jin-gu, Busan 614-735, Republic of Korea

E-mail: dyhur@inje.ac.kr

E-mail:kimdj@inje.ac.kr

Abbreviations: EBV, Epstein-Barr virus; ROS, reactive oxygen species; NAC, N-acetyl-1-cysteine

Key words: CD80, CD86, apoptosis, Epstein-Barr virus, B cells resting human B lymphocytes with EBV results in immortalization of infected B cells, yielding lymphoblastoid cells, which are characterized by continuous growth and resistance to various apoptotic signals. Moreover, lymphoblastoid cells have a phenotype related to that of activated B-lymphoblasts. However, despite clarification of the role of some viral proteins, such as latent membrane protein (LMP), in EBV-related resistance, the molecular mechanisms of resistance to apoptosis are not yet fully understood $(2,3)$.

CD40 ligand (CD40L) signals from $\mathrm{CD}^{+}{ }^{+} \mathrm{T}$ cells induce the expression of CD95 (Fas) on activated B cells that then become sensitive to the apoptotic effects of CD95 ligand (CD95L, FasL) (4). Signaling from the B cell antigen receptor (BCR) regulates whether $\mathrm{B}$ cells proliferate or undergo Fas-induced apoptosis $(5,6)$. Fas-FasL interaction plays an important role in preventing delivery of help signals from $\mathrm{CD}^{+}{ }^{+} \mathrm{T}$ cells to self-reactive B cells (7). The EBV-encoded protein LMP1 is essential for EBV-mediated B cell transformation (8). LMP1 functionally mimics the tumor necrosis factor receptor (TNFR) superfamily member CD40, although each is controlled by a different mechanism (9). EBV infection-induced LMP1 signals are amplified by both early kinase activation and downstream $\mathrm{B}$ cell effector functions and are sustained compared to CD40 (10).

Naïve resting B cells express low levels of CD86, whereas CD80 expression in these cells is either absent or present at very low levels. CD86 expression by B cells can be upregulated by triggering BCR, whereas CD80 expression can be upregulated by a variety of stimuli such as lipopolysaccharides (LPS), CD40L and many cytokines (11-15). Surface expression of the co-stimulatory molecule, CD86, is one of the early responses to BCR stimulation (16). CD86 transgenic mice that were modified to display co-stimulatory molecules on tolerant B cells showed a reversal in peripheral tolerance, T cell-dependent clonal expansion and antibody secretion (17). Several studies have reported that CD80- and CD86-mediated signaling pathways may affect $\mathrm{B}$ cell responses and the production of immunoglobulins (18-21). Moreover, triggering of CD80 specifically inhibits proliferation by upregulating the expression of pro-apoptotic molecules and downregulating levels of anti-apoptotic molecules. In contrast, CD86 enhances B cell activity (22). 
Previously, we reported that several B7 family members were upregulated by EBV infection, such as B7-H1 (PD-L1) and B7-H4. These proteins induced apoptosis both through increasing levels of reactive oxygen species (ROS) and FasL expression $(23,24)$ and reducing proliferation via cell cycle arrest at the G0-G1 phase (25). However, the role of CD80 or CD86 in EBV-transformed B cells is unclear. Thus, we aimed to explore the expression of CD86 and CD80 by EBV-transformed B-cells by investigating several known apoptosis-related events after stimulation of cells with antiCD80 or CD86 antibodies. Our results provide insight into the functions and characteristics of upregulated CD80 and CD86 on EBV-transformed B cells and can potentially be exploited for immunotherapy against malignant diseases involving EBV.

\section{Materials and methods}

Cell culture, antibodies and reagents. EBV-transformed B cells and IM-9 cells (EBV-positive human B lymphoblastoid cell line), the latter which were obtained from the American Type Culture Collection (Rockville, MD, USA), were maintained in RPMI-1640 medium (Hyclone) containing 10\% fetal bovine serum (FBS, Hyclone) and antibiotics in a $5 \% \mathrm{CO}_{2}$ atmosphere at $37^{\circ} \mathrm{C}$. Anti-CD80 (B7-1; BB1), anti-CD86 (B7-2; IT2.2), anti-Fas-PE, anti-FasL-PE, anti-CD80-FITC, antiCD86-PE, anti-CD22-FITC, anti-CD54-PE and anti-CD71-PE were purchased from BD Pharmingen (San Jose, CA, USA). Goat anti-mouse IgG, MOPC21 as a control antibody and FITC-conjugated goat anti-mouse IgG were purchased from Sigma-Aldrich (St. Louis, MO, USA). Anti-human Fas antibody ZB4 used for blocking experiments was purchased from Abcam (Cambridge, MA, USA). Mouse anti-human apoptosisinducing factor (AIF) and mouse anti-human cytochrome $c$ were purchased from Santa Cruz Biotechnologies (Santa Cruz, CA, USA). N-acetylcysteine (NAC) was purchased from Sigma-Aldrich Z-VAD-fmk ( $N$-benzyloxycarbonyl-Val-AlaAsp-fluoromethylketone) was purchased from Calbiochem (La Jolla, CA, USA). Propidium iodide (PI) was purchased from Sigma-Aldrich.

Preparation of EBV-infectious culture supernatant and generation of EBV-transformed $B$ cells. EBV stock was prepared from an EBV-transformed B95-8 marmoset-cell line (a gift from Dr B.G. Han, National Genome Research Institute, National Institute of Health, Seoul, Korea). These cells were grown in RPMI-1640 media (Hyclone, Logan, UT, USA) supplemented with $10 \%$ fetal bovine serum (Hyclone) for 7 days at $37^{\circ} \mathrm{C}$ in $5 \% \mathrm{CO}_{2}$. The culture supernatant was centrifuged $(1,000 \mathrm{rpm}$ for $10 \mathrm{~min})$, filtered using a $0.2-\mu \mathrm{m}$ pore filter (Corning, Acton, MA, USA) to remove cell debris and stored at $-80^{\circ} \mathrm{C}$ for the next experiments. To establish an EBV-infected B-cell line from normal PBMCs, $10 \mathrm{ml}$ of peripheral blood was collected from five healthy human donors (informed consent was obtained from each participant). PBMCs were isolated from whole blood by Ficoll-Paque gradient centrifugation (Amersham Biosciences, Uppsala, Sweden) and B cells were purified from PBMCs using a MACS B-cell-negative depletion kit. Purified B cells were added to B95-8 supernatant in a culture flask and after a 2-h incubation at $37^{\circ} \mathrm{C}$, an equal volume of complete medium (RPMI-1640 medium; $10 \%$ fetal bovine serum) and $1 \mu \mathrm{g} / \mathrm{ml}$ cyclosporin A (Sigma-Aldrich Inc.) was added $\left(1 \times 10^{6}\right.$ cells $\left./ \mathrm{ml}\right)$. Cultures were incubated for 2-4 weeks until clumps of EBV-infected B cells were visible and the medium turned yellow $(23,24)$. The study was approved by the Institutional Bioethics Review Board of the Medical College of Inje University and all donors provided informed consent for the study.

Detection of $B 7$ molecules (CD80 and CD86), Fas and FasL. Surface or intracellular expression of CD80 and CD86 was detected by flow cytometry or confocal laser-scanning microscopy (Carl Zeiss, 510 META, Jena, Germany). Briefly, EBV-transformed B cells or IM-9 cells were incubated with FITC-conjugated anti-CD80 or anti-CD86 antibodies and EBV-transformed B cells were permeabilized with permeabilization buffer $(0.1 \%$ saponin in PBS) to detect intracellular molecules and then stained with the same antibodies. To detect expression of Fas and FasL molecules in EBV-transformed B cells and IM-9 cells, cells stimulated with anti-CD80 or anti-CD86 antibodies were washed twice with ice-cold PBS. After two washes with ice-cold PBS, cells were incubated with mouse anti-human Fas or FasL antibodies for 30 min on ice. After two washes, cells were further incubated with the appropriate FITC-conjugated secondary antibodies in PBS for $30 \mathrm{~min}$ on ice. All samples were subjected to flow cytometry analysis using a FACSCalibur (BD Pharmingen) and data were processed using the program CellQuest (BD Pharmingen).

B7 molecule-mediated apoptosis by cross-linking or immobilization of antibodies. To immobilize anti-CD80 and CD86 $\left(\mathrm{IgG}_{1 \mathrm{k}}\right)$ or MOPC21 ( $\mathrm{IgG}_{1 \kappa}$; Sigma-Aldrich) antibodies, antibodies [50 $\mu \mathrm{g} / \mathrm{ml}$ in phosphate-buffered saline (PBS)] were coated on a 96 -well culture plate $(0.1 \mathrm{ml} /$ well; washed with PBS before use) after overnight incubation at $4^{\circ} \mathrm{C}$. EBV-transformed B cells (4 weeks, 5.0×10 ${ }^{5}$ cells/well, $200 \mu \mathrm{l}$ ) or IM-9 cells $\left(5.0 \times 10^{5}\right.$ cells/well, $\left.200 \mu \mathrm{l}\right)$ were incubated in plates coated with these antibodies at $37^{\circ} \mathrm{C}$ for $1 \mathrm{~h}$. To cross-

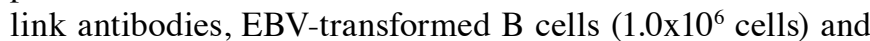
IM-9 cells $\left(1.0 \times 10^{6}\right.$ cells) were incubated in tubes with each antibody $(2 \mu \mathrm{g} / \mathrm{ml})$ at $37^{\circ} \mathrm{C}$ for $40 \mathrm{~min}$. MOPC21 $(2 \mu \mathrm{g} / \mathrm{ml})$ was used as an isotype control. Cells were washed in PBS and resuspended in $100 \mu \mathrm{l}$ of PBS and then incubated with goat anti-mouse $\mathrm{IgG}(2 \mu \mathrm{g} / \mathrm{ml})$ for $15 \mathrm{~min}$ at $37^{\circ} \mathrm{C}$. After cells were washed, they were further cultured in RPMI-1640 medium for $16 \mathrm{~h}$ at $37^{\circ} \mathrm{C}$. These cells were harvested, washed twice with PBS and then resuspended in $100 \mu \mathrm{l}$ of Annexin V-binding buffer (10 mM HEPES/NaOH pH 7.4, $140 \mathrm{mM} \mathrm{NaCl}$, $2.5 \mathrm{mM} \mathrm{CaCl} \mathrm{Ca}_{2}$ ). After $2 \mu \mathrm{l}$ of FITC-conjugated Annexin V (BD Pharmingen) was added, cells were incubated in the dark at room temperature for $15 \mathrm{~min}$ with gentle vortexing. Finally, $400 \mu \mathrm{l}$ of Annexin V-binding buffer was added to each tube and cells were analyzed using a FACSCalibur (BD Pharmingen).

Cell cycle analysis to detect a sub-G1 peak. Cellular DNA was stained with propidium iodide (PI) and quantified by flow cytometry. Briefly, EBV-transformed B cells and IM-9 cells were harvested after treatment with anti-CD80 or anti-CD86 antibodies and then washed twice in PBS (2\% FBS). After fixing the cells in $70 \%$ cold aqueous ethanol, they were stored at $4^{\circ} \mathrm{C}$ for at least $24 \mathrm{~h}$. Cells were washed twice in PBS after 

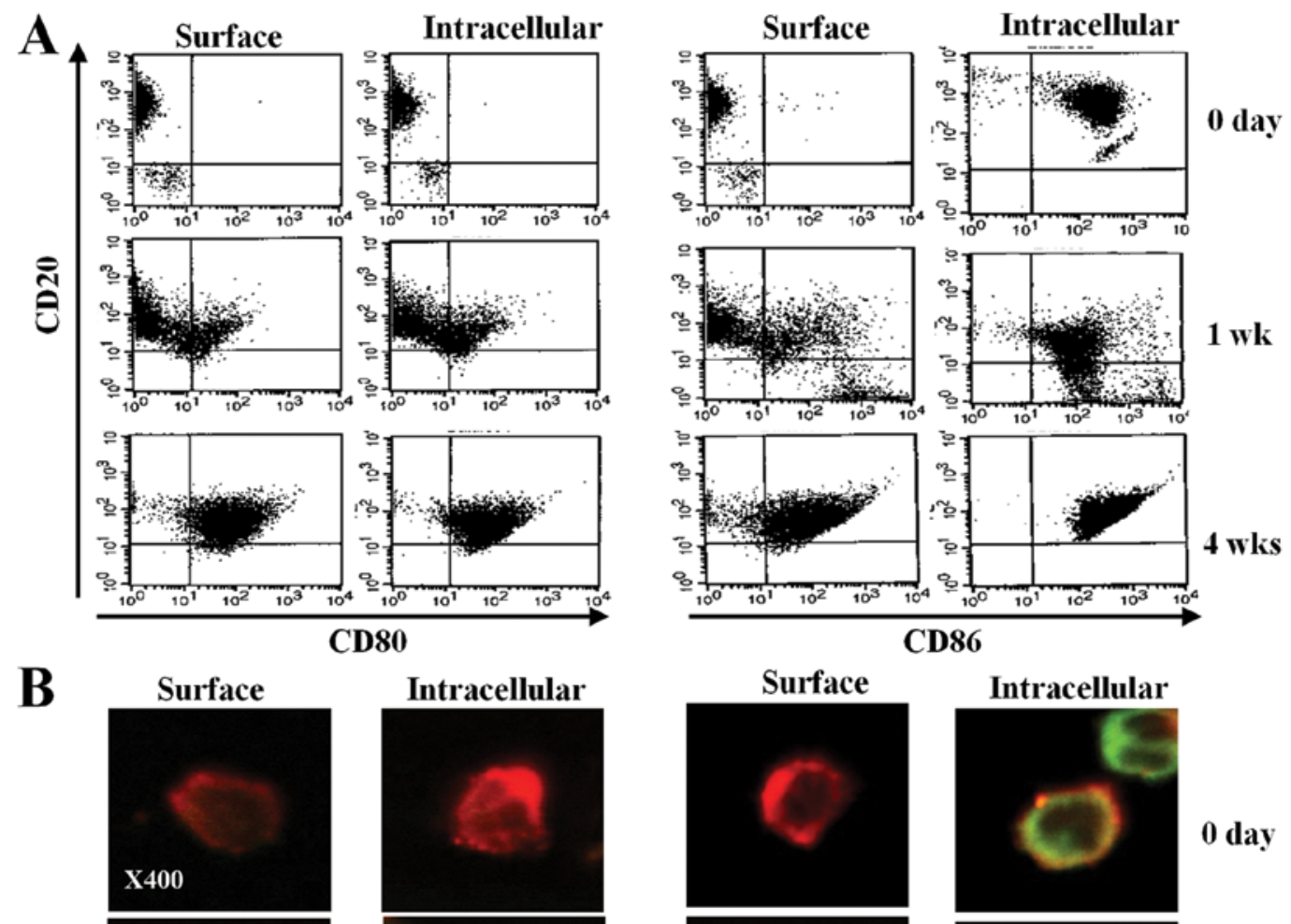

0 day
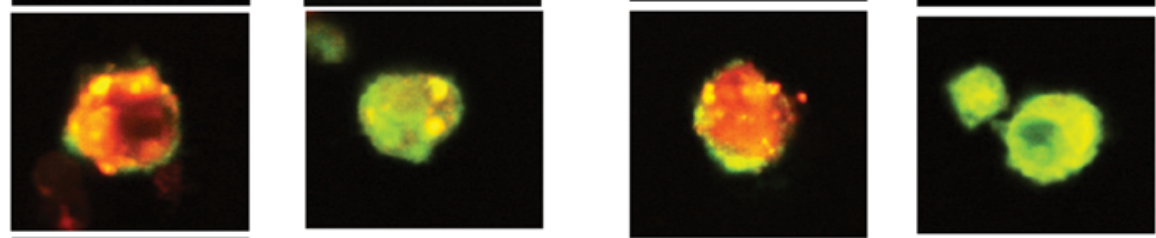

1 wk
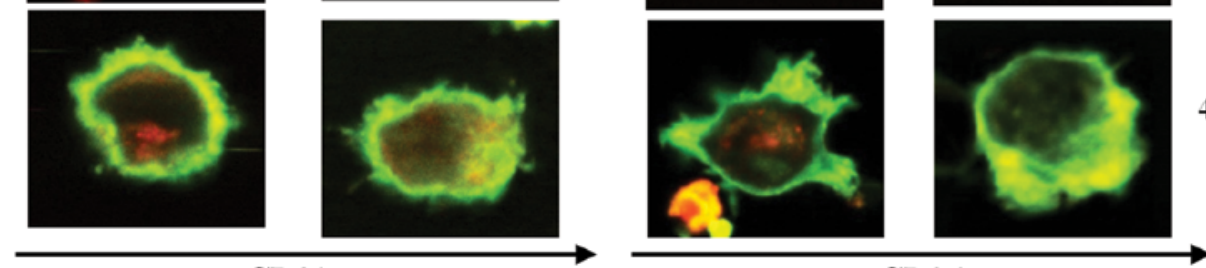

4 wks
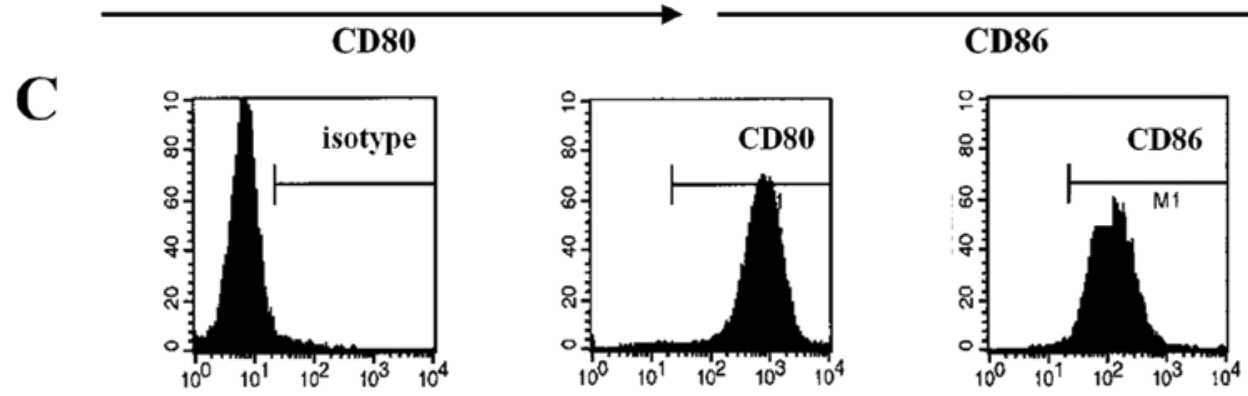

IM-9

Figure 1. CD80 and CD86 expression on EBV-transformed B cells and EBV-positive IM-9 cells. For flow cytometric analysis (A) and confocal microscopic analysis (B) of CD80 and CD86 expression on EBV-transformed B cells and IM-9 cells, cells were stained with FITC-conjugated mouse anti-human CD80 or CD86 and PE-conjugated mouse anti-human CD20, as described in Materials and methods. Confocal microscopic images show the expression of CD80 or CD86 (green) and CD20 (red) (x400). (C) EBV-positive IM-9 cells were stained with anti-CD80 or anti-CD86 or isotype control antibody. Data are representative of five independent experiments.

being centrifuged and cell pellets were stained with PI staining solution containing RNase A $(10 \mu \mathrm{g} / \mathrm{ml})$ and PI $(10 \mu \mathrm{g} / \mathrm{ml})$ in PBS. The cell suspension was then incubated in the dark at room temperature for $30 \mathrm{~min}$ and DNA content was determined using a FACSort flow cytometer (BD Pharmingen). ModFIT LT software was used for apoptotic sub-G1 peak detection (25).

Apoptosis blocking experiments. To investigate the effects of caspase inhibitors and reactive oxygen species on CD80- or
CD86-induced apoptosis, EBV-transformed B cells and IM-9 cells were pre-treated with z-VAD-fmk (20 $\mu \mathrm{M}$ in DMSO, a broad-spectrum caspase inhibitor) or NAC for $2 \mathrm{~h}$ before stimulation with antibodies. Cells were incubated with antiCD80 or anti-CD86 antibodies $(2 \mu \mathrm{g} / \mathrm{ml})$ at $37^{\circ} \mathrm{C}$ for $40 \mathrm{~min}$ followed by cross-linking with goat anti-mouse $\operatorname{IgG}(2 \mu \mathrm{g} / \mathrm{ml})$ for $15 \mathrm{~min}$ at $37^{\circ} \mathrm{C}$. The blocking effects of caspase inhibitor or NAC on apoptosis of EBV-transformed B cells and IM-9 cells were detected by Annexin $\mathrm{V}$ staining and cell cycle 

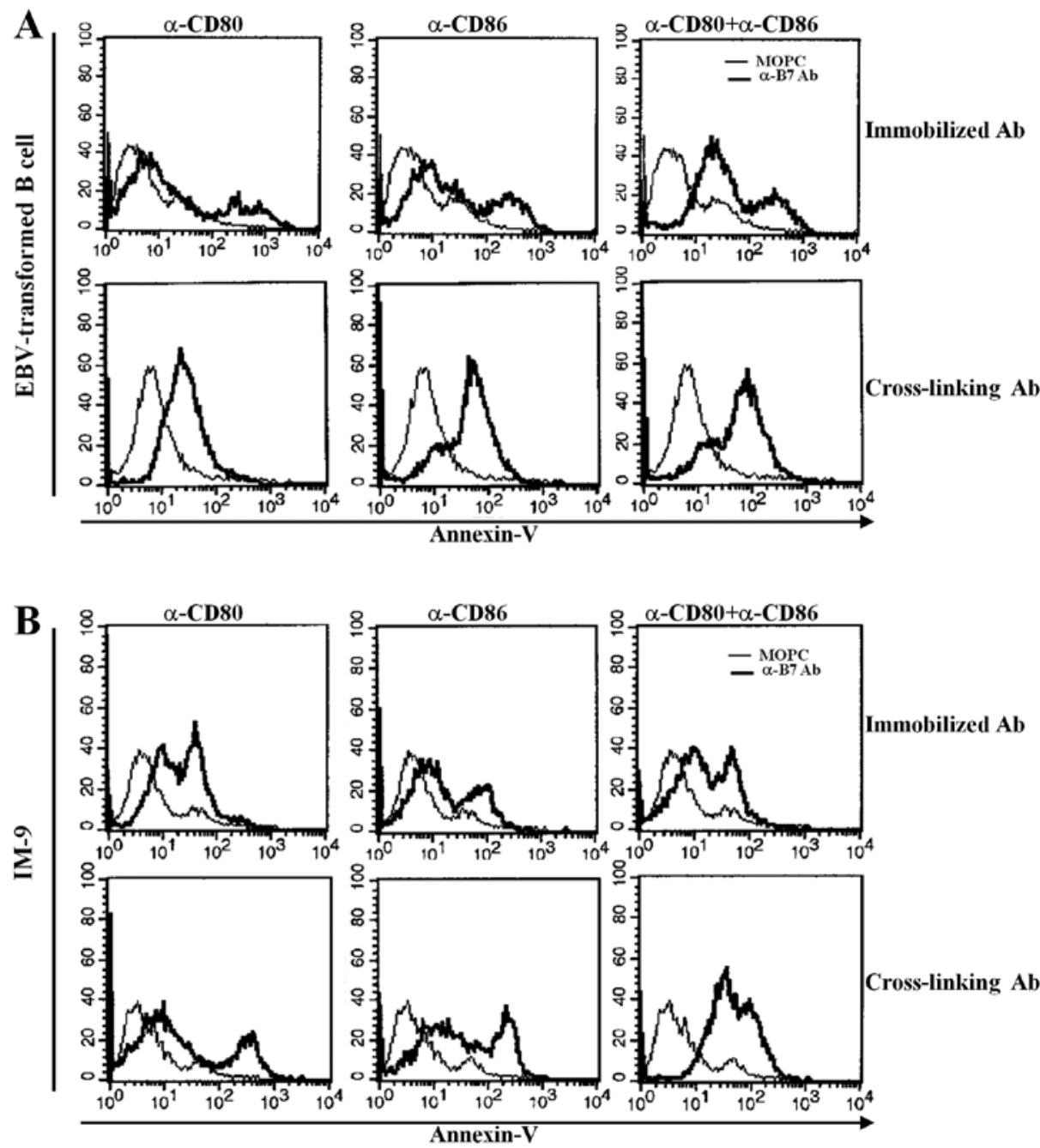

Figure 2. Engagement of CD80 and/or CD86 induces apoptosis of EBV-transformed B cells and EBV-positive IM-9 B cells. (A) EBV-transformed B cells were incubated with anti-CD80 and/or anti-CD86 antibodies as described in Materials and methods. MOPC was used as the isotype control antibody. Cells were harvested $\left(5.0 \times 10^{5}\right.$ cells for antibody immobilization; $1.0 \times 10^{6}$ cells/200 $\mu 1$ for cross-linking) and stained with FITC-conjugated Annexin V to detect apoptosis by flow cytometry. (B) EBV-positive IM-9 cells were stimulated with anti-CD80 and/or anti-CD86. MOPC was used as the isotype control antibody. Cells were harvested and stained with FITC-conjugated anti-Annexin V. Data are representative of five different experiments.

analysis as described above. To block the Fas-FasL interaction, antagonistic anti-Fas Ab ZB4 $(0.5 \mu \mathrm{g} / \mathrm{ml})$ was added $1 \mathrm{~h}$ before treatment with anti-CD80 or anti-CD86 antibodies. ZB4 was removed from cell cultures before stimulation with anti-CD80 or anti-CD86 antibodies. Apoptosis was determined by flow cytometry after staining with Annexin V.

Confocal microscopy to detect apoptosis-related intracellular molecules. EBV-transformed B cells and IM-9 cells were cross-linked with anti-CD80 or anti-CD86 antibodies followed by secondary antibodies to induce apoptosis. To detect intracellular apoptosis-related molecules, cells were permeabilized with permeabilization buffer $(0.1 \%$ saponin in PBS). Cells were incubated with primary antibodies against cytochrome $c$ (mouse IgG2b) or AIF (mouse IgG2b) and were then incubated with FITC-conjugated goat anti-mouse IgG for $30 \mathrm{~min}$. Nuclei were stained with PI for $10 \mathrm{~min}$ at room temperature. After three washes with PBS, cells were mounted on microscope slides under coverslips using a fluorescent mounting medium (Dako Cytomation, Denmark). Fluorescent cells were examined under a confocal laser-scanning microscope (Carl Zeiss,
510 META) at x400 original magnification and images were acquired using Confocal Microscopy Software Release 3.0 (Carl Zeiss, 510 META).

\section{Results}

$E B V$ transformation of primary $B$ cells increases the expression of $C D 80$ and $C D 86$. To investigate the expression of CD80 and CD86 on human B cells through EBV transformation, we observed the surface phenotype of primary $B$ cells after transformation by EBV. After EBV infection, the size of primary B cells increased and the cells clumped. Using flow cytometric analysis, we found that CD80 and CD86 were barely expressed on fresh primary B cells purified from blood (Fig 1A and B, upper panel). However, CD80 and CD86 surface expression increased on both the surface and in the cytoplasm at 1 week after EBV infection (Fig. $1 \mathrm{~A}$ and $\mathrm{B}$, middle panel). At 4 weeks after infection, most of the transformed B cells showed significantly increased expression of CD80 and CD86 (Fig. 1A and B, bottom panel). Moreover, flow cytometric data revealed upregulated expression of CD80 and CD86 molecules 


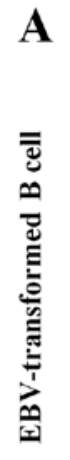
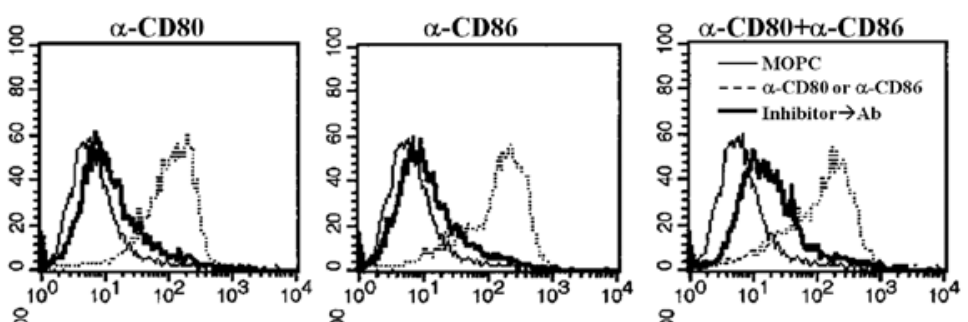

$\mathrm{Z}-\mathrm{VAD} \rightarrow \mathrm{Ab}$
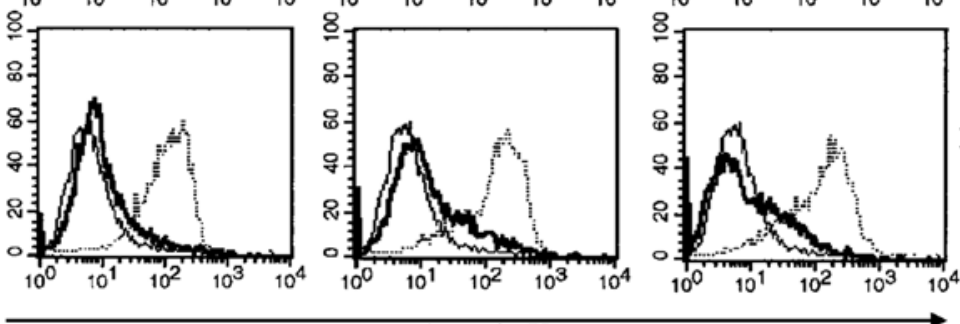

Annexin-V
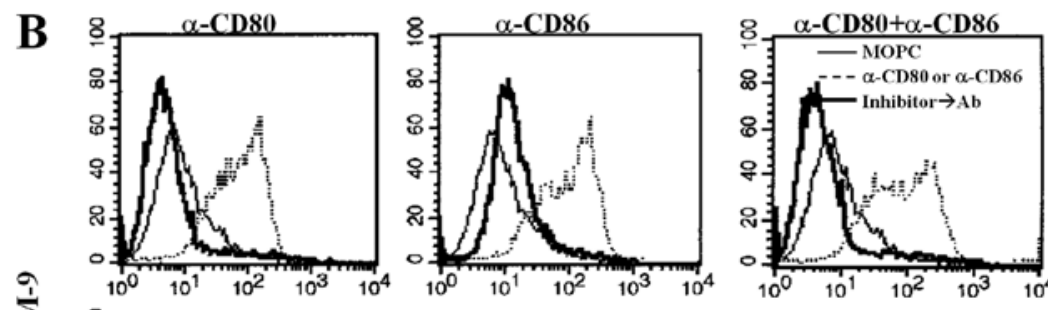

$\mathbf{N A C} \rightarrow \mathbf{A b}$
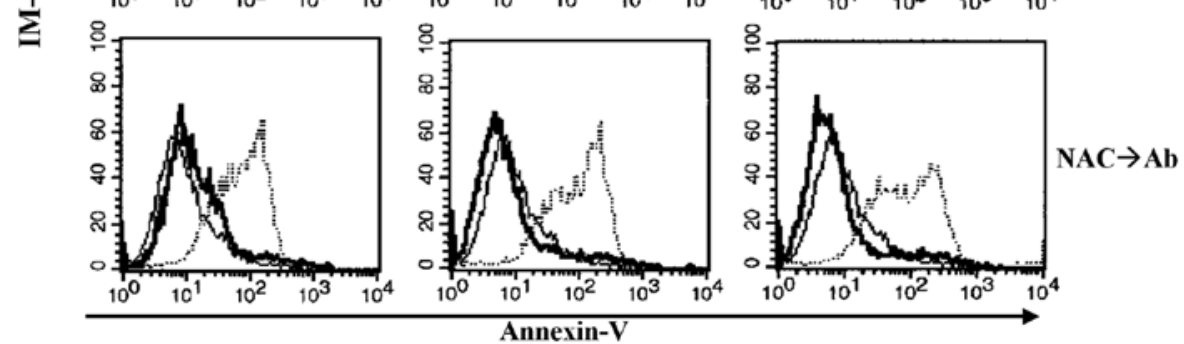

Figure 3. Effects of pancaspase inhibitor (Z-VAD) and ROS inhibitor (NAC) on CD80/86-mediated apoptosis of EBV-transformed B cells and EBV-positive IM-9 B cells. EBV-transformed B cells (A) and EBV-positive IM-9 cells (B) $\left(1 \times 10^{6}\right.$ cells $\left./ \mathrm{ml}\right)$ were preincubated with inhibitors. Cells were washed with PBS and further stimulated (5x10 cells/well, $200 \mu \mathrm{l})$ with anti-CD80 and/or anti-CD86 antibodies by cross-linking. The blocking effects of caspase inhibitor or NAC on apoptosis in EBV-transformed B cells and IM-9 cells were monitored by Annexin V staining and flow cytometry. Data are representative of three independent experiments.

on IM-9 cells, an EBV-transformed lymphoblastoid cell line (Fig. 1C). These results suggest that EBV infection induces CD80 and CD86 expression and upregulates the expression of these molecules over time.

EBV infection-induced increase in CD80 and CD86 expression induces apoptosis of both EBV-transformed B cells and IM-9 cells. Previously, we reported that other B7 family members upregulated in EBV-transformed B cells significantly induced apoptosis after stimulation with antibody $(23,24)$. EBV-transformed primary B cells and IM-9 cells were stimulated with anti-CD80 and anti-CD86-plated immobilizing antibodies for $1 \mathrm{~h}$ or were cross-linked with secondary antibody for $15 \mathrm{~min}$ to determine whether upregulation of CD80 and CD86 influenced EBV-transformed B cell apoptosis. Twenty-four hours after stimulation, cells were stained with FITC-labeled Annexin-V and were analyzed by flow cytometry. CD80 or C86 stimulation with immobilized antibodies induced apoptosis of some EBV-transformed B cells and IM-9 cells. Interestingly, co-stimulation with anti-CD80 and
CD86 antibodies anchored on the plate significantly enhanced apoptosis of EBV-transformed B cells (Fig. 2A, upper panel). Cross-linking of CD80 and CD86 effectively induced apoptosis of EBV-transformed B cells or IM-9 cells after treatment of cells with a single or both antibody types (Fig. 2A and B, lower panel). These results suggest that both CD80 and CD86 molecules are upregulated by EBV transformation and are involved in the apoptosis of EBV-transformed B cells.

CD80- and CD86-mediated apoptosis involves caspases and ROS. We examined whether CD80- and CD86-mediated apoptosis was related to caspase activity and ROS production following cross-linking with antibodies, because caspases and ROS are important mediators of apoptosis $(26,27)$. EBV-transformed B cells were pre-incubated with Z-VAD-fmk, a pan-caspase inhibitor, or NAC, a ROS inhibitor, for $2 \mathrm{~h}$ before stimulation with anti-CD80 or CD86 antibodies. Z-VAD-fmk pre-treatment blocked anti-CD80 and anti-CD86 antibodyinduced apoptosis (Fig. 3A and B, upper panel). Antioxidant treatment (NAC, $10 \mathrm{mM}$ ) also blocked the anti-CD80 and 

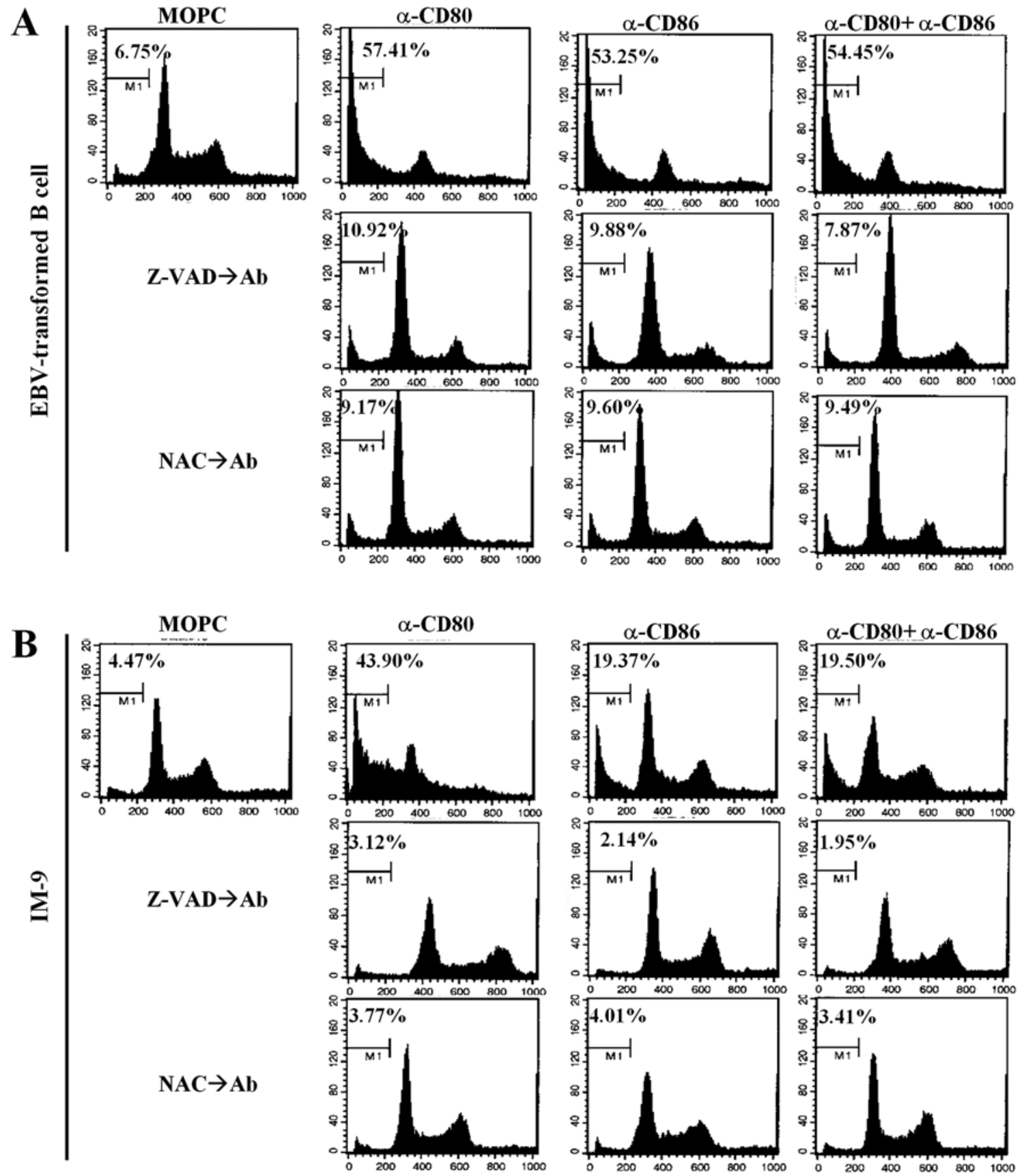

Figure 4. Effects of caspase activity and ROS on CD80/86-mediated cell cycle arrest in EBV-transformed B cells and EBV-positive IM-9 B cells. EBVtransformed B cells (A) and EBV-positive IM-9 cells (B) $\left(1 \times 10^{6}\right.$ cells $\left./ \mathrm{ml}\right)$ were preincubated with or without inhibitors (Z-VAD and NAC). Those cells were incubated in tubes with each antibody $(2 \mu \mathrm{g} / \mathrm{ml})$, washed with PBS and further incubated with goat anti-mouse $\operatorname{IgG~}(2 \mu \mathrm{g} / \mathrm{ml})$ for $15 \mathrm{~min}$ at $37^{\circ} \mathrm{C}$ for crosslinking. To evaluate sub-G1 peak apoptosis, cells were fixed in ethanol and stained with PI as described in Materials and methods. The indicated percentages represent the fraction of total cells that were sub-G1 cells. Data are representative of three independent experiments.

anti-CD86 antibody-stimulated apoptosis of EBV-transformed B cells and IM-9 cells (Fig. 3A, bottom panel).

To further elucidate the mechanisms of CD80/CD86mediated apoptosis, cell cycle analysis was performed using propidium iodide. Cross-linking of CD80 and CD86 significantly induced sub-G1 arrest in EBV-transformed B cells and the IM-9 cell line (Fig. 4A and B, upper panel). Interestingly, there was a significant increase in G0/G1 phase IM-9 cells after treatment with the anti-CD80 antibody compared to untreated cells, anti-CD86 stimulated cells and combination groups (Fig. 4B, upper panel). Pre-treatment with Z-VAD-fmk and NAC effectively restored the sub-G1 peak to the control level (Fig. 4A and B, middle and bottom panels). These results suggest that CD80- and CD86-mediated apoptosis of EBV-transformed B cells is related to ROS generation and is dependent on caspases released from mitochondria.

CD80 and CD86 stimulation of EBV-transformed B cells and IM-9 cells results in release and translocation of cytochrome $c$ and AIF from the mitochondria. Because caspases are considered to be primary apoptotic mediators $(27,28)$, we next investigated if there were changes in expression of 
A

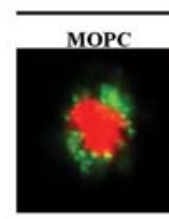

EBV-transformed B cell

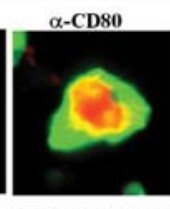

Z-VAD $\rightarrow \alpha-C D 80$
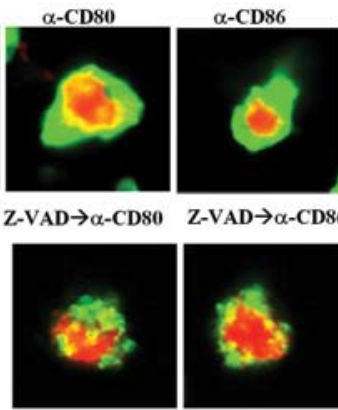

Z-VAD $\rightarrow \alpha-C D 86$
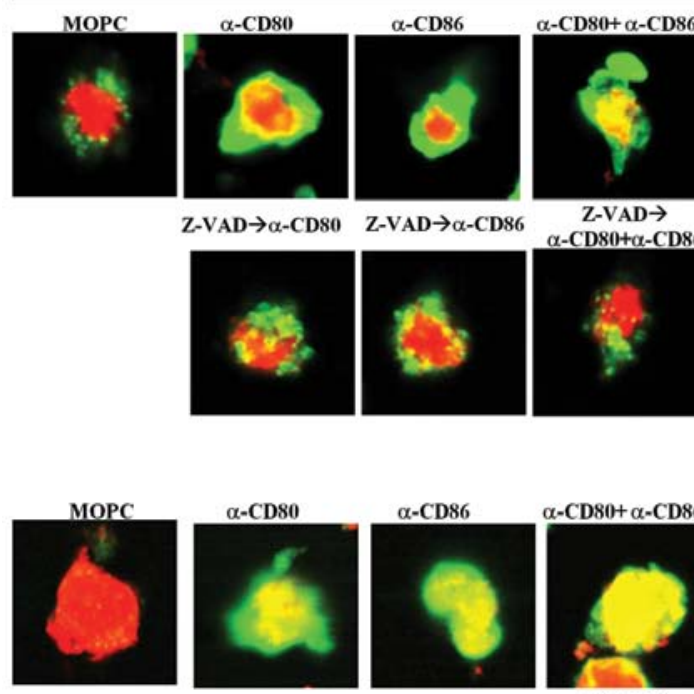

Z-VAD $\rightarrow \alpha-C D 80$
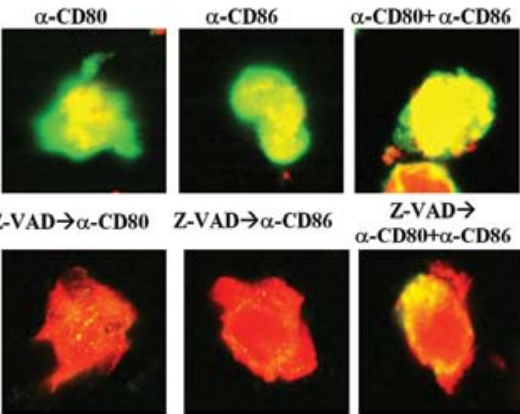

$\mathrm{Z}-\mathrm{VAD} \rightarrow$

Z-VAD $\rightarrow \alpha-C D 86$

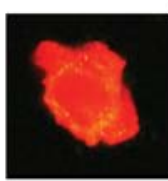

B
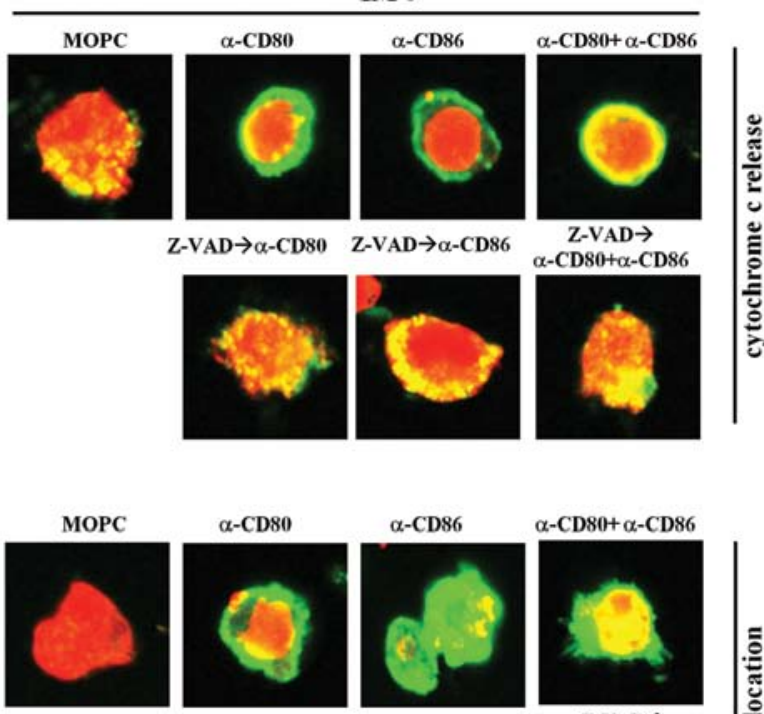

Z-VAD $\rightarrow \alpha-C D 80$

Z-VAD $\rightarrow \alpha-C D 86$
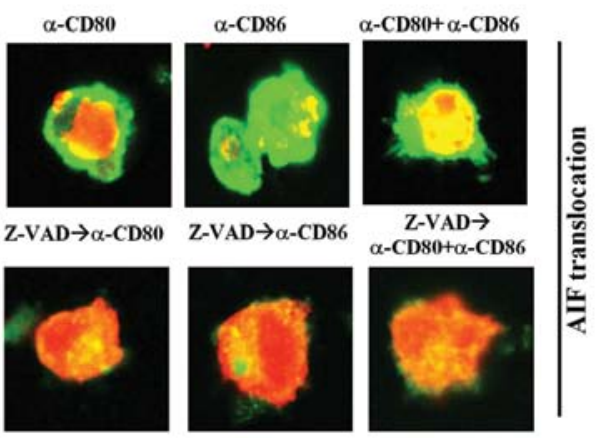

Figure 5. Subcellular distribution of cytochrome $c$ and AIF after ligation of CD80 and/or CD86 on EBV-transformed B cells and IM9 cells. EBV-transformed B cells $\left(4\right.$ weeks, $1 \times 10^{6}$ cells $\left./ 200 \mu \mathrm{l}\right)$ and IM-9 cells $\left(1 \times 10^{6}\right.$ cells/200 $\left.\mu \mathrm{l}\right)$ were stimulated with anti-CD80 and/or anti-CD86 antibodies by cross-linking. Those cells were also pre-incubated with Z-VAD-fmk $(20 \mu \mathrm{M})$ or NAC $(10 \mathrm{mM})$ for $2 \mathrm{~h}$ before stimulation. Cells were harvested and permeabilized with $0.1 \%$ saponin in PBS. Intracellular staining was performed with anti-cytochrome $c$ and AIF antibodies. FITC-conjugated secondary antibodies were used for detection. Nuclei were stained with PI (cytochrome $c$ and AIF, green fluorescence; PI, red fluorescence). Cells were observed under a confocal microscope (x400 magnification). Detailed procedures are described in Materials and methods. Data are representative of three independent experiments.

other possible apoptotic proteins, such as cytochrome $c$ and apoptosis-inducing factor (AIF), which are stored in mitochondria. We performed confocal microscopic analysis using fluorescence-conjugated secondary anti-cytochrome $c$ and anti-AIF antibodies. When stimulated with MOPC isotype control antibodies, cytochrome $c$ and AIF were localized within small mitochondria in EBV-transformed B cells and IM-9 cells (Fig. 5). In contrast, stimulation with anti-CD80 and anti-CD86 antibodies caused the release of cytochrome $c$ from mitochondria to the cytoplasm and translocation of AIF into the nucleus (Fig. 5A and B, upper panel). Moreover, we found that pre-treatment with Z-VAD-fmk and NAC largely blocked cytochrome $c$ and AIF release from the mitochondria in EBV-transformed B cells and IM-9 cells (Figs. 5A and B, lower panel). These results suggest that CD80 and CD86mediated apoptosis of EBV-transformed B cells involves mitochondria.

CD80 and CD86 stimulation induces apoptosis in EBV-transformed B cells and IM-9 cells through the Fas-FasL interaction. Because Fas/FasL can initiate apoptosis, we investigated whether stimulation of EBV-infected B cells with CD80 and CD86 changed the expression of Fas and FasL. Interestingly, CD80 and CD86 ligation of EBV-transformed B cells did not change Fas expression; Fas is constitutively expressed in EBV-transformed B cells. In contrast, CD80 and CD86 ligation significantly upregulated FasL expression (Fig. 6A). CD80/CD86-stimulation of IM-9 cells resulted in more significant induction of Fas than observed for EBV-transformed B cells and slightly increased FasL expression (Fig. 6B). To further confirm the involvement of Fas/FasL in EBV-transformed B cell apoptosis after CD80 and CD86 ligation, we examined the effect of ZB4 antibodies, which block Fas-mediated apoptosis. Pre-treatment with ZB4 antibodies for $30 \mathrm{~min}$ before cross-linking using anti-CD80 and CD86 prevented apoptosis of both EBV-transformed B cells and EBV-positive IM-9 cells (Fig. 7). These results suggest that ligation of CD80/CD86 on EBV-transformed B cells affects Fas or FasL expression and that Fas/FasL expression is closely connected with apoptosis.

\section{Discussion}

Several studies have demonstrated that lymphoblastoid cell lines (LCLs) express high levels of B-cell activation markers (CD23, CD30, CD39 and CD70) and cell adhesion molecules (29-31). These molecules are transiently induced at high levels when these cells are transformed into LCLs and the majority of them play a role in LCL proliferation. Both CD80 and CD86 are members of the immunoglobulin (Ig) super-gene family and are expressed on many cell types, including $\mathrm{T}$ cells, macrophages and dendritic cells (32-35). These molecules play a major role in the co-stimulation of T cells, subsequently leading to $\mathrm{T}$ cell-mediated immune responses, such as the killing of virus-infected cells. However, the role of CD80 and CD86 molecules induced by EBV infection of B cells has not 

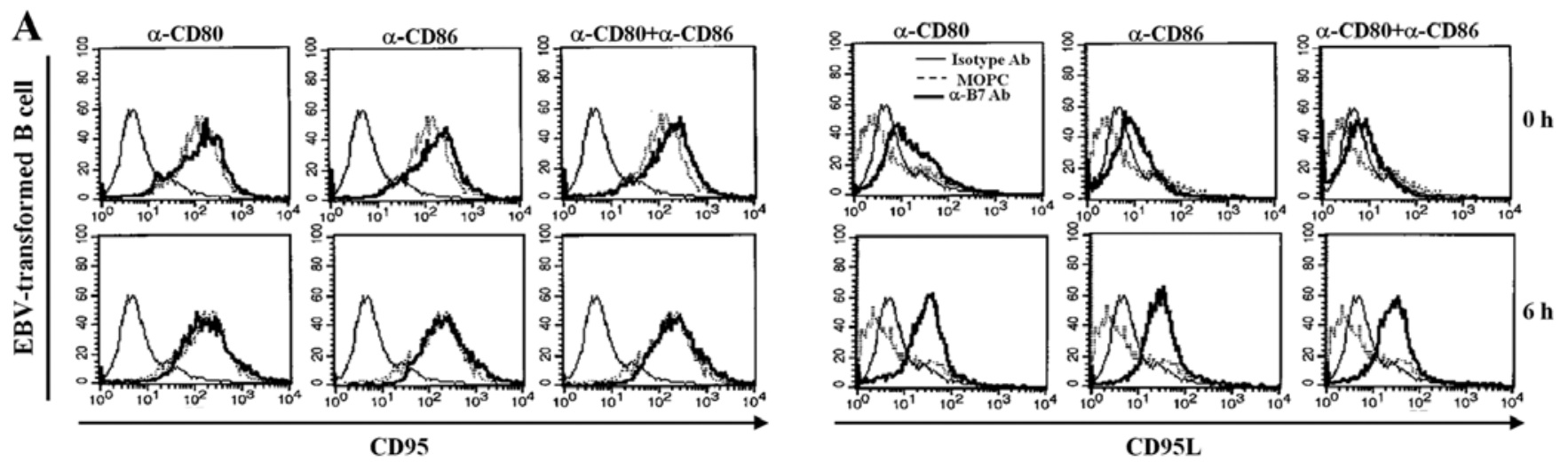

B
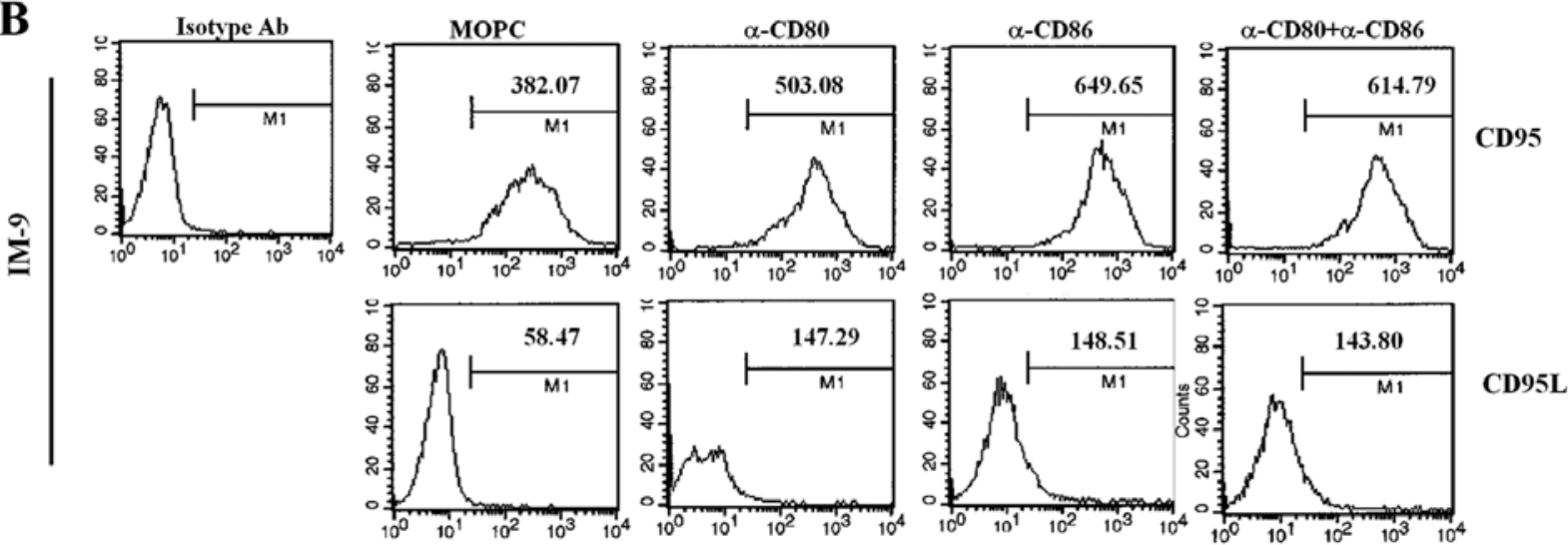

Figure 6. Fas and FasL expression on EBV transformed B cells and IM-9 cells after engagement of CD80 and/or CD86. Flow cytometric analysis was performed to detect the expression levels of CD95 (Fas) and CD176 (Fas ligand) on EBV-transformed B cells (A) and IM-9 cells (B). Cells were stained with FITC-conjugated mouse anti-human CD95 or CD176 after CD80 and CD86 stimulation with antibodies as described in Materials and methods. Data are representative of five independent experiments.

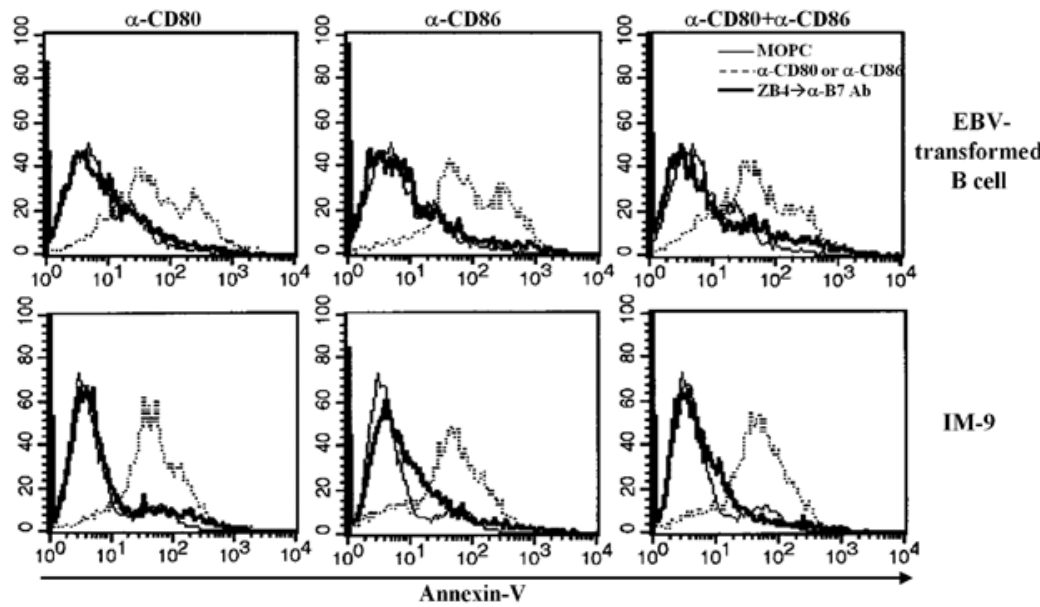

Figure 7. Effects of ZB4 (Fas-blocking antibody) on CD80/86-mediated apoptosis in EBV-transformed B cells and IM-9 cells. EBV-transformed B cells $\left(1 \times 10^{6}\right.$ cells $\left./ \mathrm{ml}\right)$ and IM-9 cells $\left(1 \times 10^{6}\right.$ cells $\left./ \mathrm{ml}\right)$ were pre-incubated with the antagonistic anti-Fas antibody, ZB4 (500 $\mu$ g/ml). Cells were washed with PBS and further stimulated with anti-CD80 and/or anti-CD86 antibodies by cross-linking. Apoptosis of those cells was detected by Annexin V staining and flow cytometry. Data are representative of three independent experiments.

been explored in previous studies. CD80 and CD86 expression by $\mathrm{B}$ cells is strongly regulated. CD80 is normally expressed at basal levels and is induced in B cells by various stimuli (e.g., cytokines, ligation of MHC class II and CD40). CD86 is constitutively expressed in B cells and is upregulated by ligation of the Ig receptor or treatment with various cytokines (11-15).

Several previous studies have reported that engagement of CD80 and CD86 by their counterpart molecules affects B cell responses and the regulation of humoral immunity $(18,20)$. 
LPS-activated B cells derived from mice expressed CD80 and the growth of activated $\mathrm{B}$ cells was regulated by upregulating the expression of caspase-3, caspase-8, Fas and FasL in the presence of anti-CD80 (16-10A1). In contrast, activation of cells via CD86 (GL1) augmented the levels of the anti-apoptotic molecules Bcl-w and $\mathrm{Bcl}-\mathrm{x}(\mathrm{L})$ and decreased the expression of caspase-8 (22). We found that both CD80 and CD86 expressed by EBV-transformed B cells derived from humans contributed to apoptosis after stimulation with antibodies. Anti-CD80 or CD86 antibodies also induced apoptosis in IM-9 cells, which are EBV-positive human B lymphoblastoid cells.

EBV-transformed B cells are known to resist Fas-mediated apoptosis due to defects in the proximal Fas signaling pathway (36) or expression of the FLICE-inhibitory protein (FLIP) (37). In contrast, another study reported that EBV-positive LCL cells established from allograft recipients were sensitive to apoptosis triggered by high-dose agonistic anti-Fas antibody and that CD95 surface expression sensitized EBV-infected B cells to the induction of CD95-mediated apoptosis $(38,39)$. ROS also regulate apoptosis signaling through the Fas and redox-sensitive transcription factors, NF- $\kappa \mathrm{B}, \mathrm{AP}-1$ and $\mathrm{p} 53$ and proinflammatory lymphokines (40). CD80 and CD86-ligation of EBV-transformed B cells resulted in the immediate production of ROS (data not shown). Stimulation of EBV-transformed B cells with CD80 or CD86 significantly increased the expression of FasL, but not Fas. In contrast, Fas and FasL expression was upregulated in IM-9 cells after stimulation with anti-CD80 or CD86 antibodies. Fas blocking antibody treatment (ZB4) successfully blocked Fas-mediated apoptosis of EBV-transformed B cells and EBV-positive IM-9 cells. This result is supported by the observation that the ROS inhibitor, NAC, not only blocked ROS generation but also prevented CD80 - and CD86-ligation-induced apoptosis. However, apoptosis induced by co-ligation of anti-CD80 and CD86 antibodies in EBV-transformed B cells was not significantly enhanced compared to control cells. Our findings indicate that CD80 and CD86 ligation of EBV-transformed B cells may contribute to the induction of apoptosis and that stimulation with CD80 and CD86 induces apoptosis of EBV-transformed lymphoblastoid B cells via the Fas/FasL pathway. However, the differences in expression of Fas and FasL in EBV-transformed B cells and IM- 9 cells after CD80 or CD86 stimulation need to be investigated.

CD80 and CD86 ligation of EBV-transformed B cells resulted in the immediate release of apoptosis-related molecules, such as cytochrome $c$ and AIF, from mitochondria. These results suggest that CD80- and CD86-mediated apoptosis may be induced through the formation of apoptosomes containing Apaf-1 and caspase-9, which ultimately activate caspase-3, an effector of apoptosis. AIF translocates directly to the nucleus and is involved in caspase-independent apoptosis $(27,28)$. Furthermore, we found that Z-VAD-fmk, a pancaspase inhibitor, interfered with CD80- and CD86-mediated apoptosis. Using confocal microscopy, we observed that cytochrome $c$ was translocated to the cytosol and AIF was translocated to the nucleus after CD80 and CD86 ligation. These results demonstrated that ligation of CD80 and CD86 induced apoptosis through caspase- and mitochondria-dependent pathways.

Taken together, our results suggest that CD80 and CD86 ligation provoke caspase-dependent apoptosis in association with cytochrome $c$ and AIF released from mitochondria in EBV-transformed B cells through the Fas-FasL interaction. This study furthers our understanding of the functions of CD80 and CD86 in activated B cells and provides basic data that can potentially be exploited to develop therapeutic options for EBV-associated cancers.

\section{Acknowledgements}

This study was supported by the 2013 Inje University Research Grant.

\section{References}

1. Epstein MA, Barr YM and Achong BG: Virus particles in cultured lymphoblasts from Burkitt's lymphoma. Lancet 15: 702-703, 1964

2. Caldwell RG, Wilson JB, Anderson SJ and Longnecker R: Epstein-Barr virus LMP2A drives B cell development and survival in the absence of normal B cell receptor signals. Immunity 9: 405-411, 1998.

3. Henderson S, Huen D, Rowe M, Dawson C, Johnson G and Rickinson A: Epstein-Barr virus-coded BHRF1 protein, a viral homologue of Bcl-2, protects human B cells from programmed cell death. Proc Natl Acad Sci USA 90: 8479-8483, 1993.

4. Rathmell JC, Townsend SE, Xu JC, Flavell RA and Goodnow CC: Expansion or elimination of B cells in vivo: dual roles for CD40and Fas (CD95)-ligands modulated by the B cell antigen receptor. Cell 87: 319-329, 1996.

5. Rathmell JC, Cooke MP, Ho WY, Grein J, Townsend SE, Davis mM and Goodnow CC: CD95 (Fas)-dependent elimination of self-reactive B cells upon interaction with $\mathrm{CD} 4^{+} \mathrm{T}$ cells. Nature 376: 181-184, 1995

6. Lagresle C, Mondière P, Bella C, Krammer PH and Defrance T: Concurrent engagement of CD40 and the antigen receptor protects naive and memory human B cells from APO-1/ Fas-mediated apoptosis. J Exp Med 183: 1377-1388, 1996.

7. Jacobson BA, Panka DJ, Nguyen KA, Erikson J, Abbas AK and Marshak-Rothstein A: Anatomy of autoantibody production: dominant localization of antibody-producing cells to T cell zones in Fas-deficient mice. Immunity 3: 509-519, 1995.

8. Arcipowski KM, Stunz LL, Graham JP, Kraus ZJ, Vanden Bush TJ and Bishop GA: Molecular mechanisms of TNFRassociated factor 6 (TRAF6) utilization by the oncogenic viral mimic of CD40, latent membrane protein 1 (LMP1). J Biol Chem 286: 9948-9955, 2011.

9. Arcipowski KM and Bishop GA: Roles of the kinase TAK1 in TRAF6-dependent signaling by CD40 and its oncogenic viral mimic, LMP1. PLoS One 7: e42478, 2012.

10. Graham JP, Arcipowski KM and Bishop GA: Differential B lymphocyte regulation by CD40 and its viral mimic, latent membrane protein 1. Immunol Rev 237: 226-248, 2010.

11. Mongini PK, Tolani S, Fattah RJ and Inman JK: Antigen receptor triggered upregulation of CD86 and CD80 in human B cells: augmenting role of the $\mathrm{CD} 21 / \mathrm{CD} 19$ co-stimulatory complex and IL-4. Cell Immunol 216: 50-64, 2002.

12. Evans DE, Munks MW, Purkerson JM and Parker DC: Resting B lymphocytes as APC for naive T lymphocytes: dependence on CD40 ligand/CD40. J Immunol 164: 688-697, 2000.

13. Clatza A, Bonifaz LC, Vignali DA and Moreno J: CD40-induced aggregation of MHC class II and CD80 on the cell surface leads to an early enhancement in antigen presentation. J Immunol 171: 6478-6487, 2003.

14. Bluestone JA: New perspectives of CD28-B7-mediated T cell costimulation. Immunity 2: 555-559, 1995.

15. Jirapongsananuruk O, Hofer MF, Trumble AE, Norris DA and Leung DY: Enhanced expression of B7.2 (CD86) in patients with atopic dermatitis: a potential role in the modulation of $\operatorname{IgE}$ synthesis. J Immunol 160: 4622-4627, 1998.

16. Lenschow DJ, Walunas TL and Bluestone JA: CD28/B7 system of T cell costimulation. Annu Rev Immunol 14: 233-258, 1996.

17. Rathmell JC, Fournier S, Weintraub BC, Allison JP and Goodnow CC: Repression of B7.2 on self-reactive B cells is essential to prevent proliferation and allow Fas-mediated deletion by CD4(+) T cells. J Exp Med 17: 651-659, 1998. 
18. Ikemizu S, Gilbert RJ, Fennelly JA, Collins AV, Harlos K, Jones EY, Stuart DI and Davis SJ: Structure and dimerization of a soluble form of B7-1. Immunity 12: 51-60, 2000.

19. Bajorath J, Peach RJ and Linsley PS: Immunoglobulin fold characteristics of B7-1 (CD80) and B7-2 (CD86). Protein Sci 3: 2148-2150, 1994.

20. Heath AW, Chang R, Harada N, Argumedo LS, Gordon J, Hannum C, Campell D, Shanafelt AB, Clark EA, Torres R and Howard M: Antibodies to murine CD40 stimulate normal B lymphocytes but inhibit proliferation of B lymphoma cells. Cell Immunol 152: 468-480, 1993.

21. Borriello F, Sethna MP, Boyd SD, Schweitzer AN, Tivol EA, Jacoby D, Strom TB, Simpson EM, Freeman GJ and Sharpe AH: B7-1 and B7-2 have overlapping, critical roles in immunoglobulin class switching and germinal center formation. Immunity 6 : 303-331, 1997.

22. Suvas S, Singh V, Sahdev S, Vohra H and Agrewala JN: Distinct role of $\mathrm{CD} 80$ and CD86 in the regulation of the activation of B cell and B cell lymphoma. J Biol Chem 277: 7766-7775, 2002.

23. Kim YS, Park GB, Lee HK, Song H, Choi IH, Lee WJ and Hur DY: Cross-linking of B7-H1 on EBV-transformed B cells induces apoptosis through reactive oxygen species production, JNK signaling activation, and fasL expression. J Immunol 181: 6158-6169, 2008

24. Song H, Park G, Kim YS, Hur I, Kim H, Ryu JW, Lee HK, Cho DH, Choi IH, Lee WJ and Hur DY: B7-H4 reverse signaling induces the apoptosis of EBV-transformed B cells through Fas ligand up-regulation. Cancer Lett 266: 227-237, 2008.

25. Park GB, Song H, Kim YS, Sung M, Ryu JW, Lee HK, Cho DH, Kim D, Lee WJ and Hur DY: Cell cycle arrest induced by engagement of B7-H4 on Epstein-Barr virus-positive B-cell lymphoma cell lines. Immunology 128: 360-368, 2009.

26. Andreyev AY, Kushnareva YE and Starkov AA: Mitochondrial metabolism of reactive oxygen species. Biochemistry 70: 200-214, 2005.

27. Saelens X, Festjens N, Vande Walle L, van Gurp M, van Loo G and Vandenabeele P: Toxic proteins released from mitochondria in cell death. Oncogene 23: 2861-2874, 2004.

28. Cregan SP, Dawson VL and Slack RS: Role of AIF in caspasedependent and caspase-independent cell death. Oncogene 23: 2785-2796, 2004.

29. Bornkamm GW and Hammerschmidt W: Molecular virology of Epstein-Barr virus. Phil Trans R Soc Lond B Biol Sci 356: 437-459, 2001.
30. Kempkes B, Spitkovsky D, Jansen-Durr P, Ellwart GW, Kremmer E, Delecluse HJ, Rottenberger C, Bornkamm GW and Hammerschmidt W: B cell proliferation and induction of early G1-regulating proteins by Epstein-Barr virus mutants conditional for EBNA2. EMBO J 14: 88-96, 1995.

31. Yamada S, Shinozaki K and Agematsu K: Involvement of CD27/ CD70 interactions in antigen-specific cytotoxic T-lymphocyte (CTL) activity by perforin-mediated cytotoxicity. Clin Exp Immunol 130: 424-430, 2002.

32. Gordon J, Millsum MJ, Guy GR and Ledbetter JA: Resting B lymphocytes can be triggered directly through the CDw40 (Bp50) antigen. A comparison with IL-4-mediated signaling. J Immunol 140: 1425-1430, 1988.

33. Goldstein MD and Watts TH: Identification of distinct domains in CD40 involved in B7-1 induction or growth inhibition. J Immunol 157: 2837-2843, 1996.

34. Nakajima A, Kodama T, Morimoto S, Azuma M, Takeda K, Oshima H, Yoshino S, Yagita $\mathrm{H}$ and Okumura K: Antitumor effect of CD40 ligand: elicitation of local and systemic antitumor responses by IL-12 and B7. J Immunol 161: 1901-1907, 1998.

35. Bergamo A, Bataille R and Pellat-Deceunynck C: CD40 and CD95 induce programmed cell death in the human myeloma cell line XG2. Br J Haematol 97: 652-655, 1997.

36. Snow AL, Chen LJ, Nepomuceno RR, Krams SM, Esquivel CO and Martinez OM: Resistance to Fas-mediated apoptosis in EBV-infected B cell lymphomas is due to defects in the proximal Fas signaling pathway. J Immunol 167: 5404-5411, 2001.

37. Tepper CG and Seldin MF: Modulation of caspase-8 and FLICEinhibitory protein expression as a potential mechanism of Epstein-Barr virus tumorigenesis in Burkitt's lymphoma. Blood 94: 1727-1737, 1999.

38. Le Clorennec C, Youlyouz-Marfak I, Adriaenssens E, Coll J, Bornkamm GW and Feuillard J: EBV latency III immortalization program sensitizes B cells to induction of CD95-mediated apoptosis via LMP1: role of NF-kappaB, STAT1, and p53. Blood 107: 2070-2078, 2006.

39. Durandy A, Le Deist F, Emile JF, Debatin K and Fischer A: Sensitivity of Epstein-Barr virus-induced B cell tumor to apoptosis mediated by anti- CD95/Apo-1/fas antibody. Eur J Immunol 27: 538-543, 1997.

40. Li-Weber $\mathrm{M}$ and Krammer PH: Function and regulation of the CD95 (APO-1/Fas) ligand in the immune system. Semin Immunol 15: 145-157, 2003. 